

\section{Student paramedic anticipation, confidence and fears: Do undergraduate courses prepare student paramedics for the mental health challenges of the profession?}

Lisa Holmes BA(Hons), is Lecturer in Paramedicine'; Russell Jones PhD, BEd(Hons), DipEd, BSc, is Professor and Lead Emergency Services Research Group ${ }^{1}$; Richard Brightwell PhD, BSc(Hons), PGCertEd, FPA, is Associate Professor $^{1}$; Lynne Cohen PhD, MPsych, BPsych, MED, BSc, is Professor and Executive Dean²

\section{Affiliations:}

${ }^{1}$ School of Medical and Health Sciences, Edith Cowan University, Perth, Western Australia

${ }^{2}$ School of Education, Edith Cowan University, Perth, Western Australia

\section{Abstract}

\section{Introduction}

This study explores the preparedness of undergraduate student paramedics for the mental health challenges of the paramedic profession from the perspective of course coordinators and their students.

\section{Methods}

Two surveys were developed and administered to course coordinators and students of the 16 undergraduate degree paramedicine courses across Australia and New Zealand. Sixteen course coordinators and 302 students responded.

\section{Results}

Results illustrate there was widespread recognition for the need to include preparation for the mental health challenges of the profession within undergraduate courses. Furthermore, most course coordinators and students had a preference for this topic to be taught using multiple teaching modes with particular preference for teaching the topic via discussion and activity based education. Teaching the topic as a standalone unit was supported by more than a third of course coordinators (43\%) and a third of students (32\%).

\section{Conclusion}

Six themes were identified as positive by anticipants: caring for people, high acuity work, diversity of work and patients, making a difference to patients and their families, using clinical skills and knowledge and engaging with the community. Students were most confident about communicating with patients and using clinical skills and knowledge. Students were least confident about clinical decision making and the most commonly cited fear was making a clinical mistake. A significant proportion of students (16\%) feared for their personal mental wellbeing and $14 \%$ reported they were least confident about personal mental health within the profession.

\footnotetext{
Keywords:

paramedic; pre-hospital; mental health; stress; depression; anxiety

Corresponding Author: Russell Jones, Russell.Jones@ecu.edu.au
} 


\section{Introduction}

Mental health challenges encountered by paramedics have received much attention in recent years. This attention has particularly focussed on high rates of stress, depression and anxiety (1). Indeed, medical and behavioural science research has established a powerful relationship between stress and psychological (as well as physical) illness (2-4). Findings that high levels of stress are present within people working in a broad spectrum of emergency service professions, and are active in the pre-hospital environment, seem to indicate there are characteristics within the pre-hospital environment rather than characteristics within individuals that lead to stress $(1,5)$. Moreover, research that reports stress is found across the paramedic profession in cultures as disparate as the Middle East (7), India (8), Japan (6) and Norway (9) as well as in western counties including Australia (10-11), the United States (12) and Canada (13) lends additional weight to the argument that stress is inherent within the pre-hospital environment rather than within individuals. Certainly there are aspects of the paramedic role that are known to contribute to the stress present in other professions. These include demanding work, poor locus of control and perceived low levels of support (14-16). Clearly the paramedic profession is a demanding profession with the need to make important decisions rapidly (ie. demanding work), while working in an unpredictable environment (ie. poor locus of control), and paramedics typically work in isolated pairs or even individually (ie. a perceived low level of support) all of which will place substantial stress on individual paramedics. This is compounded by pressures within health sectors to meet targets with a real or perceived reduction in resources together with long hours and shift work (17-18). The ultimate consequence is a stressed workforce which is costly to the individual and to the employer (1).

This heightened awareness of the high incidence of mental illness, which has at times tragically resulted in the suicide of serving and former paramedics, is stimulating the address of mental health within the paramedic profession. Indeed, there are indications the profession has one of the highest rates of work related stress, anxiety and suicide within any profession (19). Ambulance organisations have been called upon to review policy and to implement procedures to support paramedics within the workplace (20). This has commenced in a range of ways including increased wellness and peer support programs in ambulance services, as well as ongoing focussed and broad strategies such as the Ambulance Victoria Health and Safety Strategy 2016-2019 (21), Ambulance Tasmania Business Plan 2013-2016 (22), Queensland Ambulance Service Silver Linings (23), the Mental Health Reform (24) and the Wellington Free Ambulance Strategic Plan 2015 (25). There is also an opportunity to call for paramedic preparation courses to incorporate personal mental health with a view to greater preparation of graduates before commencing their careers. Although there is yet to be a formal requirement for psychological and emotional preparedness to be included within undergraduate degree programs, it would seem worthwhile to research the extent to which undergraduate paramedic education courses include aspects of mental health preparation. Mawson (26) reflects many newly qualified paramedics are filled with the excitement and anticipation of an action packed career saving lives and are not always mindful of the toll this highly rewarding career can take both physically and mentally.

There are an estimated 7484 students enrolled in undergraduate degree programs within Australia and New Zealand (27). These student paramedics are a captive audience eager to learn as much about the paramedic profession as possible. Thus, there is potential to raise awareness of the mental health challenges of the paramedic profession within a preparatory learning environment. Furthermore, there exists the opportunity in the same learning environment to teach coping strategies to meet these mental health challenges. This proactive preparatory approach promises to be far more successful than developing ad-hoc coping strategies in response to events arising within the workplace. The purpose of this research was to (a) establish current levels of preparation within undergraduate courses for the mental health challenges of the profession, (b) elicit views on the inclusion of this subject from students and course coordinators, (c) identify what students feel most and least confident as they commence their careers as paramedics, and (d) identify student anticipatory fears.

\section{Methods}

\section{Surveys}

Two qualitative surveys were constructed. The surveys were piloted on university academic staff involved in the education and training of paramedic students, practising paramedics, course coordinators and paramedic students. One survey was designed for completion by paramedicine course coordinators and focussed on the following:

1. Information about their university and enrolments

2. Their undergraduate paramedicine program and the preparation of novice paramedics for the mental health challenges of the profession

3. Course coordinator thoughts about how novice paramedics feel commencing their careers; specifically positive anticipation, confidence and fears.

They were also asked to distribute and promote completion of a student paramedic survey. 
A second survey was designed for completion by paramedicine students and focused on the following:

1. Demographic information

2. Information about their undergraduate paramedicine program and their preparation for the mental health challenges of the profession

3. Student thoughts about how they feel commencing their careers, particularly with regard to what they positively anticipate, their confidence and their fears.

Survey responses were reviewed and grouped into common themes. Themes were developed based on the responses provided by respondents. Inductive reasoning was used to develop broad themes from related specific responses. For example, when asked what students were least confident about, the responses 'making the correct diagnosis in a timecritical case' and 'taking full responsibility for the assessment, treatment and care of a patient independent from supervision of an experienced clinician' were grouped into the theme 'Clinical decision making'. Similarly, when asked what students most feared, the responses 'I am most scared of the possibility of messing up and hurting someone or killing someone' and 'making mistakes and hurting patients' were grouped into the theme 'Making a clinical mistake'.

There were strong similarities between the surveys for clinical coordinators and the survey for students. Although not identical in every respect, wherever appropriate the surveys sought to elicit responses about similar information. For example, where the course coordinator survey asked 'What do students feel most confident about when commencing their career as a paramedic?' the student survey asked 'What do you feel most confident about when commencing your career as a paramedic?' Similarly, where the course coordinator survey asked 'What do students fear the most when commencing their career as a paramedic?' the student survey asked 'What do you fear the most when commencing your career as a paramedic?' This allowed comparison between the responses of course coordinators and the responses of students.

\section{Participants}

At the time of this research in 2015 there were 16 universities offering accredited undergraduate paramedicine courses in Australia and New Zealand (27). A request for support for this research was presented to the Network of Australasian Paramedic Academics (NAPA) at the 2015 meeting. The NAPA is a 'Special Interest Group' within Paramedics Australasia which, in turn, is the peak professional association representing practitioners who provide paramedic services to the Australasian community (28-29). The NAPA agreed to support this research and surveys were sent to the undergraduate paramedicine course coordinators at each of the 16 universities. Snowball sampling (30) was used to identify undergraduate paramedicine students and included distribution of the student survey by course coordinators, dissemination of the student survey at the 2015 Paramedics Australasia International Conference, and distribution through social media. Paramedics Australasia and The Observer promoted both surveys through social media.

\section{Ethics}

Ethics approval for this study was obtained through the Edith Cowan University Human Ethics Committee: approved ethics application number 12751.

\section{Results}

Responses were obtained from 16 paramedicine undergraduate course coordinators. This represented a 100 percent response rate from course coordinators across all states and territories of Australia and New Zealand. Responses were obtained from 302 students: 100 from first year, 108 from second year, 87 from third year, and seven from the fourth year of an extended course. Some respondents chose not to answer some questions. All respondents were included within the data analysis.

\section{Preparation}

Course coordinators and students were asked whether the mental health challenges of the paramedic profession should be part of the undergraduate curriculum, does your undergraduate course currently include the preparation of students for the mental health challenges of the paramedic profession, are the mental health challenges of the paramedic profession covered in appropriate depth, and has your course suitably prepared your students/you for the mental health challenges of the profession? Responses from course coordinators and students to each of these questions are summarised in Table 1.

All course coordinators (100\%) and almost all students (97\%) reported that mental health challenges of the paramedic profession should be part of the undergraduate paramedic education and training curriculum. Three-quarters of course coordinators (75\%) and students (74\%) agreed that mental health challenges of the paramedic profession are currently included within undergraduate paramedic courses. However, there remain a significant percentage of respondents (36\% of course coordinators and $43 \%$ of students) who feel this topic is not covered in appropriate depth. Importantly, two-thirds $(65 \%)$ of course coordinators and more than half $(54 \%)$ of students reported students were not suitably prepared for the mental health challenges of the paramedic profession. Whereas the paramedic profession does not have uniform work readiness criteria against which the ability to meet mental health challenges can be measured, self-reporting based on individual paramedic personnel assessment is a powerful indicator. 
Holmes: Preparing student paramedics for mental health challenges of the profession Australasian Journal of Paramedicine: 2017;14(4)

Table 1. The proportion (\%) and number of respondents who answered yes or no for questions relating to preparation

\begin{tabular}{|l|c|c|c|c|}
\hline \multicolumn{1}{|c|}{ Question } & \multicolumn{2}{|c|}{ Course coordinators \% (N) } & \multicolumn{2}{c|}{ Students \% (N) } \\
\cline { 2 - 5 } & Yes & No & Yes & No \\
\hline $\begin{array}{l}\text { Should the mental health challenges of the paramedic } \\
\text { profession be part of the undergraduate curriculum? }\end{array}$ & $100 \%(15)$ & $0 \%(0)$ & $97 \%(274)$ & $3 \%(8)$ \\
\hline $\begin{array}{l}\text { Does your undergraduate course currently include the } \\
\text { preparation of students for the mental health challenges of the } \\
\text { paramedic profession? }\end{array}$ & $75 \%(12)$ & $25 \%(4)$ & $74 \%(225)$ & $26 \%(78)$ \\
\hline $\begin{array}{l}\text { Are the mental health challenges of the paramedic profession } \\
\text { covered in appropriate depth? }\end{array}$ & $64 \%(7)$ & $36 \%(4)$ & $57 \%(113)$ & $43 \%(84)$ \\
\hline $\begin{array}{l}\text { Has your course suitably prepared your students/you for the } \\
\text { mental health challenges of the profession? }\end{array}$ & $35 \%(5)$ & $65 \%(9)$ & $46 \%(113)$ & $54 \%(131)$ \\
\hline
\end{tabular}

Table 2. The proportion (\%) and number of respondents reporting how preparation for mental health challenges is taught and how it should be taught

\begin{tabular}{|l|c|c|c|c|}
\hline \multirow{2}{*}{ Mode of instruction } & \multicolumn{2}{|c|}{ Course coordinators \% (N) } & \multicolumn{2}{c|}{ Students \% (N) } \\
\cline { 2 - 5 } & $\begin{array}{c}\text { How is the material } \\
\text { taught? }\end{array}$ & $\begin{array}{c}\text { How should the } \\
\text { material be taught? }\end{array}$ & $\begin{array}{c}\text { How is the } \\
\text { material taught? }\end{array}$ & $\begin{array}{c}\text { How should the } \\
\text { material be taught? }\end{array}$ \\
\hline Lecture & $100 \%(11)$ & $86 \%(12)$ & $81 \%(162)$ & $69 \%(171)$ \\
\hline Discussion & $100 \%(11)$ & $93 \%(13)$ & $63 \%(126)$ & $81 \%(202)$ \\
\hline Activity & $82 \%(9)$ & $86 \%(12)$ & $36 \%(73)$ & $65 \%(162)$ \\
\hline Independent research & $9 \%(1)$ & $21 \%(3)$ & $34 \%(68)$ & $26 \%(65)$ \\
\hline Group research & $36 \%(4)$ & $64 \%(9)$ & $9 \%(19)$ & $24 \%(61)$ \\
\hline A standalone unit & $45 \%(5)$ & $43 \%(6)$ & $24 \%(48)$ & $32 \%(79)$ \\
\hline Placement/practicum & $19 \%(1)$ & $7 \%(1)$ & $9 \%(19)$ & $11 \%(27)$ \\
\hline
\end{tabular}

Course coordinators and students were asked how the topic of mental health challenges of the paramedic profession is taught and how it should be taught within the undergraduate curriculum. Responses from course coordinators and students are summarised in Table 2.

Most course coordinators and students reported mental health challenges in the paramedic profession were addressed using multiple teaching modes and most had a preference that this topic be taught in multiple teaching modes. Whereas lectures were the most common way the topic was addressed, both course coordinators and students reported a preference for teaching the topic via discussion. Teaching the topic through activity based education was also a preference for both course coordinators (86\%) and students (65\%). Teaching the topic as a standalone unit was supported by more than a third of course coordinators (43\%) and almost a third $(32 \%)$ of students. 
There was significant support by course coordinators for teaching the topic through group research (64\%) and some support by students (24\%). There was some support by both course coordinators $(21 \%)$ and students $(26 \%)$ for teaching the topic through independent research. There was little support for teaching the topic as placement/practicum by either course coordinators or students.

\section{Positive anticipation}

Course coordinators and students were asked what students most look forward to about commencing paramedicine as a career. This question elicited six themes: caring for people, high acuity work, diversity of work and patients, making a difference to patients and their families, using clinical skills and knowledge, and engaging with the community. Responses from course coordinators and students to each of these themes are summarised in Table 3.

Close to a third of students (30\%) and a quarter of course coordinators $(25 \%)$ reported caring for people was the greatest positive anticipation for students prior to commencing a career as a paramedic. Conversely, half of course coordinators (50\%) and almost a fifth of students (18\%) reported the prospect of high acuity work was most anticipated. A third (33\%) of course coordinators and almost an eighth (11\%) of students reported making a difference to patients and their families was most anticipated. The diversity of work and patients was considered an area of positive anticipation for $13 \%$ of students and $17 \%$ of course coordinators. Perhaps surprisingly, given the extent of the investment in undergraduate courses of teaching clinical skills and knowledge, the use of clinical skills and knowledge was not cited by any course coordinators and only $9 \%$ of students as the most anticipated positive theme. Engagement with the community was considered the most positively anticipated response by only $17 \%$ of course coordinators and $9 \%$ of students.

\section{Confidence (most)}

Course coordinators and students were asked 'what do students feel most confident about when commencing their/ your career as a paramedic?' This question elicited nine themes: communication with patients, using clinical skills and knowledge, working in a team, commitment to the profession, being able to keep calm, building a rapport with patients, feeling passionate about my job, having support from colleagues, and those who had not yet developed strong confidence. Responses from course coordinators and students to each of these themes are summarised in Table 4.

More than a quarter $(26 \%)$ of students reported they felt most confident about communicating with patients yet no course coordinators felt students were most confident with this theme. More than half of course coordinators (64\%) and a fifth of students $(21 \%)$ reported being most confident about using clinical skills and knowledge. Almost a fifth (18\%) of course coordinators and $11 \%$ of students felt they were most confident in working as a team and in their commitment to the profession. Slightly fewer students (8\%) cited being able to keep calm, $6 \%$ cited building a rapport with patients and still fewer (5\%) cited feeling passionate about their job. Seven students (3\%) and one course coordinator $(9 \%)$ reported students did not yet feel sufficiently confident to identify a theme. Other low volume responses included attending mentally ill patients and having the physical strength required to undertake the role.

Table 3. The themes, proportion (\%) and number of respondents who identified each theme when asked what students most look forward to about commencing paramedicine as a career

\begin{tabular}{|c|c|c|c|}
\hline Question & Themes & Course coordinators \% $(\mathrm{N})$ & Students \% (N) \\
\hline \multirow{6}{*}{$\begin{array}{l}\text { What do students/did you } \\
\text { look forward to most about } \\
\text { commencing their/your career } \\
\text { as a paramedic? }\end{array}$} & Caring for people & $25 \%(3)$ & $30 \%(72)$ \\
\hline & High acuity work/career & $50 \%(6)$ & $18 \%(42)$ \\
\hline & Diversity of work and patients & $17 \%(2)$ & $13 \%(31)$ \\
\hline & $\begin{array}{l}\text { Making a difference to patients and } \\
\text { their families }\end{array}$ & $33 \%(4)$ & $11 \%(26)$ \\
\hline & Using clinical skills/knowledge & - & $9 \%(22)$ \\
\hline & Engaging with the community & $17 \%(2)$ & $9 \%(21)$ \\
\hline
\end{tabular}


Holmes: Preparing student paramedics for mental health challenges of the profession Australasian Journal of Paramedicine: 2017;14(4)

Table 4. The themes, proportion (\%) and number of respondents who identified each theme when asked what students were most confident about

\begin{tabular}{|l|l|c|c|}
\hline \multirow{2}{*}{ Question } & \multicolumn{1}{|c|}{ Themes } & $\begin{array}{c}\text { Course coordinators } \\
\%(\mathrm{~N})\end{array}$ & Students \% (N) \\
\hline \multirow{4}{*}{$\begin{array}{c}\text { What do students/you feel most } \\
\text { confident about when commencing } \\
\text { their/your career as a paramedic? }\end{array}$} & Communication with patients & - & $26 \%(59)$ \\
\cline { 2 - 4 } & Using clinical skills and knowledge & $64 \%(7)$ & $21 \%(48)$ \\
\cline { 2 - 4 } & Working in a team & $18 \%(2)$ & $11 \%(24)$ \\
\cline { 2 - 4 } & Bemmitment to the profession & - & $1 \%(24)$ \\
\cline { 2 - 4 } & Building a rapport with patients & - & $8 \%(18)$ \\
\cline { 2 - 4 } & Feeling passionate about my job & - & $6 \%(14)$ \\
\cline { 2 - 4 } & Not yet confident & $9 \%(1)$ & $3 \%(7)$ \\
\cline { 2 - 4 } & Having support from colleagues & $9 \%(1)$ & - \\
\hline
\end{tabular}

Table 5. The themes, proportion (\%) and number of respondents who identified each theme when asked what students were least confident about

\begin{tabular}{|c|c|c|c|}
\hline Question & Themes & $\begin{array}{c}\text { Course coordinators } \\
\%(\mathrm{~N})\end{array}$ & Students \% (N) \\
\hline \multirow{15}{*}{$\begin{array}{l}\text { What do students feel least } \\
\text { confident about when commencing } \\
\text { their/your career as a paramedic? }\end{array}$} & Clinical decision making & $45 \%(5)$ & $26 \%(56)$ \\
\hline & Personal mental health well being & - & $14 \%(32)$ \\
\hline & Working with children & $18 \%(2)$ & $9 \%(21)$ \\
\hline & Getting a job & - & $8 \%(17)$ \\
\hline & $\begin{array}{l}\text { Dealing with aggressive intoxicated } \\
\text { patients }\end{array}$ & - & $7 \%(15)$ \\
\hline & Understanding other cultures & - & $7 \%(15)$ \\
\hline & Working with mental health patients & $9 \%(1)$ & $5 \%(12)$ \\
\hline & $\begin{array}{l}\text { Communication with ESOL patients } \\
\text { and families }\end{array}$ & - & $5 \%(12)$ \\
\hline & Dealing with the death of a patient & - & $4 \%(8)$ \\
\hline & Adapting to shift work & - & $4 \%(8)$ \\
\hline & Complex cases & $27 \%(3)$ & - \\
\hline & Driving an ambulance in bad weather & $9 \%(1)$ & - \\
\hline & Being in charge of a case & $9 \%(1)$ & - \\
\hline & Putting practice into reality & $9 \%(1)$ & - \\
\hline & Being able to act quickly & $9 \%(1)$ & - \\
\hline
\end{tabular}


Holmes: Preparing student paramedics for mental health challenges of the profession Australasian Journal of Paramedicine: 2017;14(4)

\section{Confidence (least)}

Course coordinators and students were asked 'what do students feel least confident about when commencing their/ your career as a paramedic?' This question elicited almost twice as many themes (15) as the question regarding what they feel most confident. These themes were: clinical decision making, personal mental health wellbeing, working with children, getting a job, dealing with aggressive intoxicated patients, understanding other cultures, working with mental health patients, communication with patients and families for whom English is not their primary language, dealing with the death of a patient, adapting to shift work, complex cases, driving an ambulance in bad weather, being in charge of a case, putting practice into reality and being able to act quickly. Responses from course coordinators and students to each of these themes are summarised in Table 5.

Clinical decision-making was identified by nearly half of course coordinators (45\%) and a quarter of students $(25 \%)$ as the area of least confidence upon commencing a career as a paramedic. There was some agreement between course coordinators (18\%) and students (10\%) that working with children was an area of least confidence. Students also noted that personal mental health wellbeing was an area of least confidence (14\%). Course coordinators $(27 \%)$ and no students noted complex cases as an area of least confidence. Getting a job (8\%), dealing with aggressive patients (7\%) and understanding other cultures (7\%) were each cited by students though no course coordinators reported these themes. Similarly, communication with English for speakers of other languages (ESOL) patients and families $(5 \%)$, dealing with the death of a patient (4\%) and adapting to shift work (4\%) were reported by students but not by course coordinators. Conversely, a third of all themes were reported by course coordinators but not students. These were complex cases $(27 \%)$, driving an ambulance in bad weather $(9 \%)$, being in charge of a case (9\%), putting practice into reality (9\%) and being able to act quickly (9\%). One course coordinator and 12 students cited working with mental health patients as an area of least confidence. Other low volume responses included: wearing the uniform, knowing when a patient can be left at home and issues relating to law and ethics.

\section{Fears}

Course coordinators and students were asked what students fear about commencing paramedicine as a career. This question elicited 10 themes: making a clinical mistake, personal mental wellbeing, not getting a job, treating children, aggressive and abusive patients, the death of a patient, multiple casualties, working with unsupportive colleagues, being accepted as an equal and motor vehicle accidents. Responses from course coordinators and students to each of these themes are summarised in Table 6.

Table 6. The themes, proportion (\%) and number of respondents who identified each theme when asked what students fear the most when commencing their career as a paramedic

\begin{tabular}{|c|c|c|c|}
\hline Question & Themes & Course coordinators \% (N) & Students \% (N) \\
\hline \multirow{10}{*}{$\begin{array}{l}\text { What do students/you fear the } \\
\text { most when commencing their/ } \\
\text { your career as a paramedic? }\end{array}$} & Making a clinical mistake & $36 \%(4)$ & $27 \%(62)$ \\
\hline & Personal mental well being & - & $16 \%(37)$ \\
\hline & Not getting a job & $9 \%(1)$ & - \\
\hline & Treating children & - & $9 \%(21)$ \\
\hline & Aggressive and abusive patients & - & $8 \%(18)$ \\
\hline & The death of a patient & - & $6 \%(13)$ \\
\hline & Multiple casualties & $9 \%(1)$ & $3 \%(8)$ \\
\hline & $\begin{array}{l}\text { Working with unsupportive } \\
\text { colleagues }\end{array}$ & $18 \%(2)$ & $2 \%(4)$ \\
\hline & Being accepted as an equal & $9 \%(1)$ & $0.5 \%(1)$ \\
\hline & Motor vehicle accidents & $9 \%(1)$ & - \\
\hline
\end{tabular}


Making a clinical mistake received the most responses from both course coordinators (36\%) and students (27\%). Although $16 \%$ of students feared for their personal mental wellbeing, no course coordinators noted this as a fear. Conversely, $18 \%$ of course coordinators reported students feared working with unsupportive colleagues yet only $2 \%$ of students agreed. Fears identified by students but not course coordinators included treating children (9\%), aggressive and abusive parents $(8 \%)$ and death of a patient $(6 \%)$. Small numbers of course coordinators and students cited multiple casualties ( $9 \%$ and $3 \%$ ) and being accepted as an equal ( $9 \%$ and $0.5 \%$ ). Not getting a job and motor vehicle accidents were each identified as the most feared event for students by one course coordinator but not by students. Other fears reported by students included litigation, lack of experience, facing death on a regular basis, getting injured and not measuring up to paramedic expectations.

\section{Discussion}

The 100 percent response rate from undergraduate paramedicine course coordinators is indicative of the interest the paramedic education and training community has in mental health issues and the importance this community places on this topic.

\section{Preparation}

All course coordinators (100\%) and almost all (97\%) students believed mental health challenges within the paramedic profession should be part of the undergraduate paramedic education and training curriculum. This highlights the perceived importance of pro-actively addressing the mental health challenges of paramedicine. Moreover, this result supports the inclusion of this topic within the learning phase.

Reports by three-quarters of both course coordinators $(75 \%)$ and students $(74 \%)$ revealed agreement that the paramedic profession's mental health challenges are currently included within undergraduate paramedic courses. This shows that despite there being no formal requirement to include preparation for the mental health challenges of the profession, most courses have at least included some level of provision. Anecdotal reports reveal some courses have only recently included mental health challenges of the paramedic profession. However, this is interpreted as a growing awareness amongst paramedic educators about the value of this topic and part of the ongoing evolution of optimal education and training courses to meet the needs of their students and the profession.

Importantly, a significant percentage of both course coordinators $(36 \%)$ and students $(43 \%)$ reported mental health challenges within paramedicine were not covered in appropriate depth within their courses. Moreover, two-thirds $(65 \%)$ of course coordinators reported students were not suitably prepared for the mental health challenges of the paramedic profession. These are pertinent responses and demonstrate a need for additional inclusion of educational content in this area. It may also be argued that if inclusion of personal and colleague mental health and wellbeing were a formal requirement in the accreditation of courses, additional resources maybe allocated to this area by education providers as part of the accreditation process. Paramedic education and training curricula are crowded with many valuable topics. Course coordinators may have difficulty in meeting competing demands when designing courses. Further, there may be resourcing issues that limit the time and resources spent on this topic. Importantly more than half $(54 \%)$ of students reported they will not be suitably prepared for mental issues in the paramedic profession.

It is noteworthy that when course coordinators were asked if students were adequately prepared for the mental health challenges of the paramedic profession, only $25 \%$ responded in the affirmative whereas $75 \%$ responded in the negative. This thinking by course coordinators as they reflect on their own paramedic experiences may explain why they have sought to include mental health preparation within undergraduate paramedicine courses.

While it is encouraging that there exists agreement between course coordinators and students about the need to teach this topic there was much variety in how the topic was taught with very little specific formal content being dedicated within course units. While the inclusion of the topic in a lecture format was a popular choice for both course coordinators and students, the opportunity for discussion and activity was also seen as advantageous. Independent research would serve to raise awareness, but further engagement in collaboration with others would encourage open conversation. It is worth considering the use of a range of learning opportunities throughout the course that would normalise this topic and encourage the consideration of personal coping strategies and how to support each other. From a pedagogical perspective it is best to teach preparation for meeting the mental health challenges of the paramedic profession using multiple teaching methods. This is because it is a recognised pedagogical principle that teaching using multiple modes ensures optimum learning for cohorts of students who will have disparate and varied learning styles (31).

\section{Positive anticipation}

That almost a third of students (30\%) and a quarter (25\%) of course coordinators reported caring for people was the greatest positive anticipation for students before commencing a career as a paramedic aligns with the first of the top 10 reasons to become a paramedic listed by the New South Wales Ambulance Service (32). The finding that more than a half of course coordinators (50\%) and almost a fifth of students (18\%) reported the prospect of high acuity work was most anticipated clearly reveals high acuity work is considered important. 
This response was noted in ways such as the use of lights and sirens, the urgency of a situation and the excitement of trauma calls. The difference between the findings reported by course coordinators and students may be due to the greater knowledge and experience of course coordinators compared with novice students. Given the nature of service to the community within paramedicine it was pleasing to note that making a difference to patients and their families was most anticipated by $33 \%$ of course coordinators and $11 \%$ of students. Respondents phrased these responses in different words including: helping people on their worst day, caring for people in their darkest hour and making a bad situation better.

\section{Confidence (most)}

Of the nine identified themes in which students had most confidence, there was agreement between course coordinators on two: using clinical skills and knowledge, and working as a team. Whereas concurrency of reporting for these two themes reveals common views by both course coordinators and students, four themes were only reported by students. These were commitment to the profession, being able to keep calm, building a rapport with patients and feeling passionate about their job. All of these are beneficial and valuable characteristics of paramedics. Perhaps understandably, small numbers of course coordinators and students, primarily the most novice, did not yet feel sufficiently confident to identify a theme. The agreement between course coordinators and students regarding student ability to use clinical skills and knowledge and to work as a team is particularly pleasing given these are key aspects for successful paramedics. Being confident about working as a team also suggests interpersonal support and mentorship are highly valued. It is also possible this suggests students see value in their communication skills which have been honed within their courses, placements, volunteer work, employment and domestic lives.

\section{Confidence (least)}

Clinical decision making was the most identified area of least confidence for students by both course coordinators and students. It may be students are comfortable with what they have learned yet need support and validation from others to make final decisions. Students noted that personal mental health wellbeing was an area of least confidence for $14 \%$ of responses. This may be a result of the national (33) and international (34) media attention focussing on mental health within paramedicine as well as heightened awareness due to the survey used in this study. Interestingly, course coordinators reported five areas of least student confidence that were not reported by students. These were complex cases, driving an ambulance in bad weather, being in charge of a case, putting practice into reality and being able to act quickly. This is likely to be a consequence of course coordinators having significantly greater paramedic experience than students, and therefore course coordinators having a broader understanding of specific challenges that await graduating students. Getting a job was also mentioned by students $(8 \%)$, which shows awareness that there are limited positions in Australia. That more students did not focus on employment issues as an area of least confidence may be explained by students being confident in their ability to find employment or by their being least confident in other areas. Other low volume responses included wearing the uniform, knowing when a patient can be left at home, and law and ethics.

\section{Fears}

A sixth (16\%) of students most feared for their personal mental wellbeing. As noted above, this may be a consequence of current media attention focussing on mental health within paramedicine as well as heightened awareness caused by this study. Alternatively, this finding may indeed reflect increasing self-awareness within students about the demands of paramedicine and human psychological frailty. Making a clinical mistake received the most responses from both course coordinators (36\%) and students (27\%). Given the clinical nature of paramedicine work, plus the potential negative consequences of making a clinical error, this fear is likely understandable in novice paramedics. It is even possible that fear of making a clinical error may serve as a motivating factor among many novice paramedics to be clinically vigilant. That almost a fifth (18\%) of course coordinators reported students feared working with unsupportive colleagues (with only $2 \%$ of students agreeing) may be reflective of the course coordinators' own experiences as novice paramedics or an awareness of the importance of supportive colleagues for novice paramedics. Not getting a job, multiple casualties, being accepted as an equal and motor vehicle accidents were each reported as most feared by a clinical coordinator, but not by students. This is likely to be a consequence of the greater experience of course coordinators within the profession of paramedicine that provides clinical coordinators with a different perspective to students.

\section{Conclusion}

The mental health challenges of the paramedic profession have received considerable attention in recent years. Whereas there has been anecdotal evidence that this topic is valued by those involved in the education and training of novice paramedics, as well as the novice paramedics themselves and paramedic employers, this study is the first to research this topic among undergraduate course coordinators and students. The $100 \%$ response rate to the course coordinator survey and the completion of student surveys from all undergraduate degree programs across Australia and New Zealand during the study period is strongly indicative of the importance with which stress within paramedicine is viewed. 
The negative effects of stress, anxiety and depression are well known. Stress has been documented in a number of emergency service professions and has been found to occur within paramedicine across a range of nationalities and cultures. Thus it is seems likely that characteristics within the pre-hospital environment, rather than characteristics within individuals, lead to stress. Therefore educational programs that are directed to all paramedics will be most successful in supporting paramedics to meet the mental health challenges of paramedicine. In particular, the undergraduate education programs that provide the qualifications of choice to Australian and New Zealand employers. These programs are rich and fertile environments in which to begin to prepare novice paramedics to meet the mental health challenges of paramedicine. Whereas undergraduate teaching cannot, and must not, replace ongoing programs and initiatives by paramedic organisations intended to support mental health, inclusion of teaching designed to foster the ability of novice paramedics to successfully meet the challenges of the paramedic profession does offer a valuable opportunity to proactively address the challenges that arise in the prehospital environment.

There is value in promoting undergraduate degree programs to include comprehensive preparation for the mental health challenges of the profession, to raise awareness of mental health in paramedicine, and to educate student paramedics before commencing their careers. Student paramedics are a captive audience and there is potential to raise awareness of the mental health challenges of the paramedic profession within their preparatory learning environments. Furthermore, there exists the opportunity in the same learning environments to teach coping strategies to meet the mental health challenges.

\section{Acknowledgements}

The authors would like to acknowledge the support of all undergraduate course coordinators and students who completed surveys as part of this research. The authors would also like to thank members of the Network of Australasian Paramedic Academics, Paramedics Australasia and The Observer who facilitated distribution of the surveys and data collection.

\section{Conflict of interest}

The authors declare they have no competing interests. Each author of this paper has completed the ICMJE conflict of interest statement.

\section{References}

1. Mildenhall J. Occupational stress, paramedics informal coping strategies: a review of the literature. Journal of Paramedic Practice 2012;4:318-26.

2. Cooper CL. Handbook of stress medicine and health. 2nd edn. London: CRC; 2005. p. 440.

3. Sinha BK, Watson DC. Stress, coping and psychological illness: a cross-cultural study. Int J Stress Manag 2007;14:386-97.

4. Tosevski DL, Milovancevic MP. Stressful life events and physical health. Curr Opin Psychiatr 2006;19:184-9.

5. Hancock PA, Desmond PA. Stress, workload, and fatigue. Mahwah NJ: Lawrence Erlbaum; 2001.

6. Okada N, Noboru Ishii N, Nakata M. Nakayama S. Occupational stress among Japanese emergency medical technicians: hyogo prefecture. Prehosp Disaster Med 2005;20:115-21.

7. Heydari A, Rad M, Ghasemi MR, Sabzevari MT, Rad $M$. Challenges ahead of emergency medical technician graduates in the workplace in Iran: a qualitative study. J Allied Health 2014;43:e19-24.

8. Khashaba E, El-Sherif M, Adel Ibrahim A, Neatmatallah M. Work-related psychosocial hazards among emergency medical responders (EMRS) in Mansoura city. Indian J Comm Med 2014;39:103.

9. Sterud T, Hem E, Ekeberg O, Lau B. Occupational stressors and its organsisational and individual correlates: a nationwide study of Norwegian ambulance personnel. BMC Emerg Med 2008;8:1-11.

10. Maguire B, O'Meara P, O'Neill B, Fitzgerald J, Brightwell R. Occupational injury risk among Australian paramedics: an analysis of national data. Med J Aust 2014;200:477-80.

11. Dollard MF, LaMontagne AD, Caulfield N, Blewett V, Shaw A. Job stress in the Australian and international health and community services sector: a review of the literature. Int J Stress Manag 2007;14:417-45.

12. Bentley MA, Crawford JM, Wilkins JR, Fernandez AR, Studnek JR. An assessment of depression, anxiety, and stress among nationally certified EMS professionals. Prehosp Emerg Care 2013;17:330-8.

13. Donnelly EA, Bradford P, Davis M, Hedges C, Klingel M. Predictors of posttraumatic stress and preferred sources of social support among Canadian paramedics. Can J Emerg Med 2016;18:205-12.

14. de Lange AH, Taris TW, Kompier MAJ, Houtman ILD, Bongers PM. The very best of the millennium: longitudinal research and the demand-control-(support) model. J Occup Health Psychol 2003;8:282-305. 


\section{References (continued)}

15. Regehr C, Millar D. Situation critical: low control, and low support in paramedic organisations. Traumatology 2007;13:49-58.

16. van Vegchel N, de Jonge J, Bosma H, Schaufeli W. Reviewing the effort-reward imbalance model. Soc Sci Med 2005;60:1117-31.

17. Jenner $M$. The psychological impact of responding to agricultural emergencies. Aust J Emerg Manag 2007;22:2531.

18. Nirel N, Goldwag R, Feigenberg Z. Stress, work overload, burnout and satisfaction among paramedics in Israel.

Prehosp Disaster Med 2008;23:537-44.

19. Dangerous jobs: The project (television broadcast). Melbourne: Network Ten; 16 September 2014.

20. Powell G. Report of suicides prompts union call for more counselling for paramedics (document on the Internet). $A B C$ News; 25 November 2014. Available at: www.abc.net.au/ news/2014-11-25/paramedics-suffering-from-ptsd-needmore-counselling-union-say/5916932 [Accessed 20 August 2016].

21.Ambulance Victoria Health and Safety Strategy 2016-2019 (document on the internet). Ambulance Victoria. Available at: http://s3-ap-southeast-2.amazonaws.com/prod.assets. ambulance.vic.gov.au/wp-content/uploads/2016/06/ ambulance-victoria-health-safety-strategy-2016-2019.pdf [Accessed 20 August 2016].

22. Ambulance Tasmania Business Plan 2013-2016 (document on the internet). Ambulance Tasmania. Available at: www.dhhs.tas.gov.au/ambulance/welcome to_tasmanian_ambulance_and_health_transport_service/ publications?a=164806 [Accessed 30 August 2016].

23. Murray J. Finding the silver lining: stress, resilience and growth in ambulance practice. Brisbane, Australia. Queensland Ambulance Service, 2013.

24. Mental health reform - unfinished business (document on the internet). Australian Health Care Reform Alliance. Available at: www.healthreform.org.au/wp-content/uploads/2016/05/ MRMentalHealth27May16.pdf [Accessed 20 August 2016].

25. Wellington Free Ambulance Strategic Plan 2015. We are the ONES here for you (document on the internet). Wellington Free Ambulance. Available at: www.wfa.org.nz/file/661/ Wellington-Free-Ambulance-Strategic-Plan-2015.pdf [Accessed 30 August 2016].

26. Mawson C. Broken: A Paramedic's Battle with PTSD. Perth, Australia Kindle, 2015. p. 160.

27. CAA accredited courses. Council of Ambulance Authorities. Available at: www.caa.net.au/paramedic-education/ accredited-courses [Accessed June 2015].

28. About NAPA. Paramedics Australasia. Available at: www. paramedics.org/our-organisation/special-interest-groups/ network-of-australasian-paramedic-academics/about-napa/ [Accessed 15 August 2016].

29.Paramedics Australasia. Available at: www.paramedics.org/ our-organisation/who-we-are/ [Accessed 15 August 2016].

30. Goodman LA. Snowball sampling. Annals of Mathematical Statistics 1961;32:148-70.

31. Jones $R$. The influence of student learning styles and faculty teaching preferences on medical school approaches to problem-based learning. J Med Sci 2007;27:189-96.

32. Top ten reasons to become a paramedic. Ambulance Service of New South Wales. Available at: hwww. ambulance.nsw.gov.au/Employment/Paramedic-Positions/ Top-five-reasons-to-become-a-paramedic.html [Accessed 20 August 2016].

33. Knowles $L$. New figures reveal high suicide rates amongst emergency workers; experts warn PTSD sufferers not getting needed treatment. ABC National News; 4 June 2015. Available at: www.abc.net.au/news/2015-06-03/ new-figures-reveal-high-suicide-rates-amongst-emergencyworkers/6518250 [Accessed 9 January 2017].

34. We paramedics save lives but NHS cuts have stretched us to breaking point. The Guardian, London UK, 6 February 2015. Available at: www.theguardian.com/ commentisfree/2015/feb/05/paramedics-save-lives-nhs-cutsbreaking-point [Accessed 9 January 2017]. 